\title{
Degradation of the cancer genomic DNA deaminase APOBEC3B by SIV Vif
}

\author{
Allison M. Land ${ }^{1,2}$, Jiayi Wang ${ }^{1}$, Emily K. Law ${ }^{1}$, Ryan Aberle $^{1}$, Andrea Kirmaier ${ }^{3}$, \\ Annabel Krupp ${ }^{3,4}$, Welkin E. Johnson ${ }^{3}$, Reuben S. Harris ${ }^{1}$ \\ ${ }^{1}$ Department of Biochemistry, Molecular Biology and Biophysics, Institute for Molecular Virology, Masonic Cancer Center, and \\ Center for Genome Engineering, University of Minnesota, Minneapolis, Minnesota, USA \\ ${ }^{2}$ Present address: Department of Biological Sciences, Minnesota State University Mankato, Mankato, Minnesota, USA \\ ${ }^{3}$ Department of Biology, Boston College, Boston, Massachusetts, USA \\ ${ }^{4}$ Present address: Biogen Idec, Cambridge, Massachusetts, USA \\ Correspondence to: \\ Reuben S. Harris, e-mail: rsh@umn.edu \\ Keywords: $A P O B E C 3 B$, cancer mutagenesis, endogenous DNA deamination, lentiviral Vif, tumor evolution \\ Received: June 07, 2015 \\ Accepted: October 19, 2015 \\ Published: October 31, 2015
}

\section{ABSTRACT}

\begin{abstract}
APOBEC3B is a newly identified source of mutation in many cancers, including breast, head/neck, lung, bladder, cervical, and ovarian. APOBEC3B is a member of the APOBEC3 family of enzymes that deaminate DNA cytosine to produce the promutagenic lesion, uracil. Several APOBEC3 family members function to restrict virus replication. For instance, АPOBEC3D, АPOBEC3F, АPOBEC3G, and APOBEC3H combine to restrict HIV-1 in human lymphocytes. HIV-1 counteracts these APOBEC3s with the viral protein Vif, which targets the relevant APOBEC3s for proteasomal degradation. While АРОВЕСЗВ does not restrict HIV-1 and is not targeted by HIV-1 Vif in CD4-positive $T$ cells, we asked whether related lentiviral Vif proteins could degrade АРОВЕСЗВ. Interestingly, several SIV Vif proteins are capable of promoting AРОВЕСЗВ degradation, with SIVmac239 Vif proving the most potent. This likely occurs through the canonical polyubiquitination mechanism as APOBEC3B protein levels are restored by MG132 treatment and by altering a conserved E3 ligase-binding motif. We further show that SIVmac239 Vif can prevent APOBEC3B mediated geno/cytotoxicity and degrade endogenous АРОВЕСЗB in several cancer cell lines. Our data indicate that the APOBECЗB degradation potential of SIV Vif is an effective tool for neutralizing the cancer genomic DNA deaminase APOBEC3B. Further optimization of this natural APOBEC3 antagonist may benefit cancer therapy.
\end{abstract}

\section{INTRODUCTION}

The DNA cytosine deaminase APOBEC3B (A3B) was identified recently as a major source of mutation in cancer [1-11]. A3B was initially determined to be upregulated in breast tumors, and this upregulation correlates with increased mutation loads at its preferred DNA deamination motif (i.e. 5'-TC-3') [1]. These mutations have been observed to occur in clusters, termed kataegis, and correlated with translocations and other chromosomal aberrations $[6,12,13]$. Since these findings, A3B has been further implicated in contributing to the mutational load in breast cancer and other malignancies, including bladder, cervical, head/neck, lung, and ovarian cancers [2-11]. Furthermore, clinical data have begun to accumulate, demonstrating that elevated $\mathrm{A} 3 \mathrm{~B}$ expression correlates with poor outcomes in breast cancer patients $[14,15]$. Together, these studies support a model in which A3B is a major source of mutation in cancer that drives tumor evolution, therapy resistance, and poor patient outcomes (reviewed in [16-18]).

$\mathrm{A} 3 \mathrm{~B}$ is part of the seven-membered APOBEC3 family of proteins, which share the ability to deaminate DNA cytosine to uracil - a pro-mutagenic base. The physiologic roles of the family members are antiviral immunity. However, each APOBEC3 appears specialized to restrict certain pathogens. For example, four members; APOBEC3D (A3D), APOBEC3F (A3F), APOBEC3G 
(A3G), and $\mathrm{APOBEC} 3 \mathrm{H}(\mathrm{A} 3 \mathrm{H})$, have the ability to restrict the lentivirus human immunodeficiency virus-1 (HIV-1) in $\mathrm{T}$ lymphocytes by catalyzing mutations in the viral genome and interfering with reverse transcription (many labs, reviewed in $[19,20])$. HIV-1 counteracts restriction by binding these APOBEC3 enzymes with the virally encoded Vif protein, and targeting them for E3 ubiquitin ligase-mediated proteasomal degradation (reviewed in [19, 20]). The human A3B (huA3B) protein, however, does not restrict HIV-1 in T cells, and is not neutralized by HIV-1 Vif [21-28].

Most lentiviruses in addition to HIV-1 encode a Vif protein, including simian immunodeficiency virus (SIV), bovine immunodeficiency virus (BIV), feline immunodeficiency virus (FIV), and maedi-visna virus (MVV). The Vif proteins from these viruses also function to degrade the cognate restrictive $\mathrm{APOBEC} 3$ proteins from each mammalian host [22, 29-32]. The number and type of APOBEC 3 proteins that are encoded by each animal host can vary, with simians expressing seven APOBEC3 proteins (similar to humans), cats expressing five APOBEC 3 proteins, and cows and sheep each expressing four APOBEC3 proteins [33-35]. It is generally believed that each Vif protein has undergone evolutionary optimization to specifically degrade the APOBEC 3 s of each host. However, cross-species degradation has been documented and indeed likely occurs to allow zoonotic transmission [32, 35, 36]. Based on this rationale, we hypothesized that at least one naturally occurring lentiviral Vif would have human A3B (huA3B) antagonizing activity.

To test this idea, we surveyed a panel of Vif proteins from diverse lentiviruses (see Methods for a full listing of viral isolates) and found that SIVmac239 Vif is a potent neutralizer of huA3B, while several other SIV Vif proteins are also capable of promoting huA3B degradation. MG132 treatment inhibited degradation, as did altering the conserved E3 ligase-binding motif, indicating that the degradation likely occurs through the established polyubiquitination mechanism. Finally, we demonstrated that SIVmac239 Vif can prevent huA3B mediated geno/ cytotoxicity and degrade endogenous huA3B in multiple human cancer cell lines. Our studies thereby establish SIVmac239 Vif as a molecular tool that may be further developed into a therapeutic strategy to counteract huA3B, decrease tumor mutation rates, and improve patient outcomes.

\section{RESULTS}

\section{SIVmac239 Vif triggers huA3B degradation}

We and others have previously demonstrated that HIV-1 ${ }_{\text {ІІІв }}$ Vif does not efficiently mediate degradation of huA3B during viral infection [21-25, 37]. To determine huA3B sensitivity to degradation by various lentiviral Vif proteins, we tested the ability of a panel of Vif constructs derived from HIV-1 ${ }_{\text {IIIIB }}$, SIVmac239, BIV, MVV, and FIV to mediate degradation of huA3B. These Vif constructs were transfected into $293 \mathrm{~T}$ cells at near-equivalent levels based on immunoblots, along with a constant amount of huA3B or vector control (Fig. 1A). The expected sizes of these Vif proteins range from approximately $23.9 \mathrm{kDa}$ for HIV- ${ }_{\text {IIII }}$ Vif to $30.4 \mathrm{kDa}$ for FIV Vif including the $1.2 \mathrm{kDa}$ carboxy-terminal MYC epitope tag. HIV- $1_{\text {ІІІв }}$ Vif demonstrated inefficient counteraction of huA3B, as it was only able to mediate degradation of huA3B at the highest expression levels. SIVmac239 Vif was the most efficient at mediating degradation of huA3B, with the lowest level of SIVmac239 Vif mediating a similar level of huA3B degradation as the highest level of HIV-1 ${ }_{\text {IIIB }}$ Vif. Furthermore, the highest level of SIVmac239 Vif rendered huA3B barely detectable by immunoblot (Fig. 1A). HuA3B cotransfected with BIV Vif showed moderately lower levels of expression regardless of the amount of BIV Vif co-transfected. FIV Vif and MVV Vif did not have any effect on huA3B, regardless of expression levels.
A

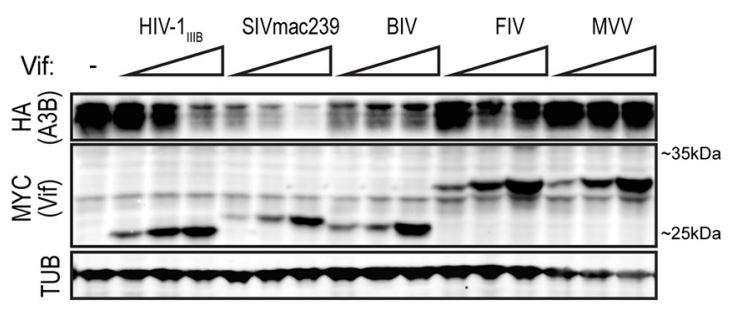

B

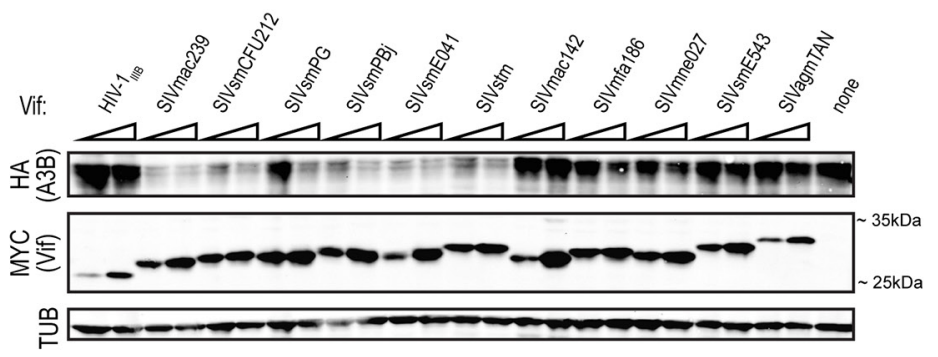

Figure 1: SIVmac239 Vif efficiently degrades huA3B. A. Immunoblot demonstrating the varying abilities of the lentiviral Vif proteins to degrade huA3B. The lysates were blotted for MYC to detect Vif, HA to detect huA3B, and tubulin (TUB) as a loading control. B. Representative immunoblot demonstrating the abilities of Vif from HIV-1 ${ }_{\text {IIIB }}$ and the indicated SIV isolates to degrade huA3B. The lysates were blotted for MYC to detect Vif, HA to detect huA3B, and tubulin (TUB) as a loading control. The migration positions of molecular weight standards are indicated next to the anti-MYC (Vif) panels. 
To determine if degradation of huA3B is a property elicited by Vif from multiple SIV strains, we tested a diverse panel of SIV Vif expression constructs. The panel consisted of lentiviruses that naturally infect sooty mangabeys, rhesus macaques, stump-tailed macaques, pigtailed macaques, cynomolgus macaques, and African green monkeys [38]. As described above, these Vif constructs were expressed in 293T cells at near-equivalent levels based on immunoblots, with huA3B or vector control, and cellular lysates were probed to assess the level of Vif-mediated degradation of huA3B (Fig. 1B). Many of the different SIV Vif proteins caused degradation of huA3B. These include Vif from SIVmac239, SIVsmCFU212, SIVsmPG, SIVsmPBj, SIVsmE041, and SIVstm. Additionally, many of the different SIV Vif proteins were unable to mediate degradation of huA3B. These Vif proteins include SIVmac142, SIVmfa186, SIVmne027, SIVsmE543, SIVagmTAN, as well as Vif from HIV-1 (Fig. 1B). Over multiple independent experiments, SIVmac239 Vif consistently expressed well and elicited a strong huA3B degradation phenotype. For these reasons, additional experiments focused on SIVmac239 Vif.

\section{SIVmac239 Vif degrades huA3B in a manner analogous to HIV-1 ${ }_{\text {IIIB }}$ Vif degradation of huA3G}

To determine if SIVmac239 Vif mediates degradation of huA3B in an analogous manner to HIV-1 Vif degradation of huA3G, which has been studied extensively (reviewed in $[19,20]$ ), we tested for Vifmediated relief of $\mathrm{HIV}-1$ restriction in single-cycle infectivity assays. Additionally, we examined rhesus macaque A3B (rhA3B) susceptibility to SIVmac239 Vif, as this protein is the cognate target of SIVmac239 Vif. HuA3B, rhA3B, huA3G, and vector control constructs were transfected into the $293 \mathrm{~T}$ cell line with Vif-deficient full-length molecular clone $\mathrm{HIV}-1_{\mathrm{IIIB}}$. Another vector control, HIV-1 $1_{\text {IIIB }}$ Vif, or SIVmac239 Vif were cotransfected into the cells on separate expression vectors. As shown previously, huA3G restricted viral infectivity in the absence of any Vif protein, but lesser so when HIV-1 ${ }_{\text {IIIB }}$ Vif was present (Fig. 2A). The ability of huA3G to restrict HIV replication was even more strongly counteracted by SIVmac239 Vif, as reported [39, 40]. Overall, both HIV$1_{\text {IIIB }}$ and SIVmac239 Vif proteins have the capacity to degrade huA3G (Fig. 2B). In contrast, huA3B restricted HIV-1 $1_{\text {IIIB }}$ infectivity both in the absence of Vif protein and in the presence of HIV-1 ${ }_{\text {IIIB }}$ Vif. Only SIVmac239 Vif was able to relieve huA3B-mediated restriction of HIV-1 (Fig. 2A), and only SIVmac239 Vif promoted degradation of huA3B (Fig. 2B). The rhA3B protein showed a similar restriction profile to huA3B. RhA3B was restrictive in the absence of Vif, and in the presence of HIV-1 ${ }_{\text {IIIB }}$ Vif. SIVmac239 Vif moderately restored viral infectivity in the presence of rhA3B (Fig. 2A), and had a minor effect on rhA3B degradation (Fig. 2B), as reported [22, 32].
To further characterize similarities between huA $3 \mathrm{G}$, huA3B, and rhA3B counteraction by HIV-1 ${ }_{\text {IIIB }}$ Vif and SIVmac239 Vif, we tested whether the observed degradation occurs through a ubiquitin-mediated proteasomal degradation pathway, as is the case for HIV1 Vif-mediated degradation of huA $3 \mathrm{G}$, by inhibiting proteasomal degradation with the compound MG132 $[41,42]$. As expected, MG132 inhibited degradation of huA3G by HIV-1 ${ }_{\text {IIIB }}$ Vif and SIVmac239 Vif (Fig. 3A). SIVmac239 Vif, but not HIV-1 ${ }_{\text {IIIB }}$ Vif, mediated degradation of huA3B, and this degradation was also inhibited by MG132. RhA3B was somewhat degraded in the presence of SIVmac239 Vif, while HIV-1 ${ }_{\text {IIIB }}$ Vif was not observed to mediate degradation of rhA3B. Inhibition of the proteasome with MG132 decreased SIVmac239 Vif mediated degradation of rhA3B (Fig. 3A).

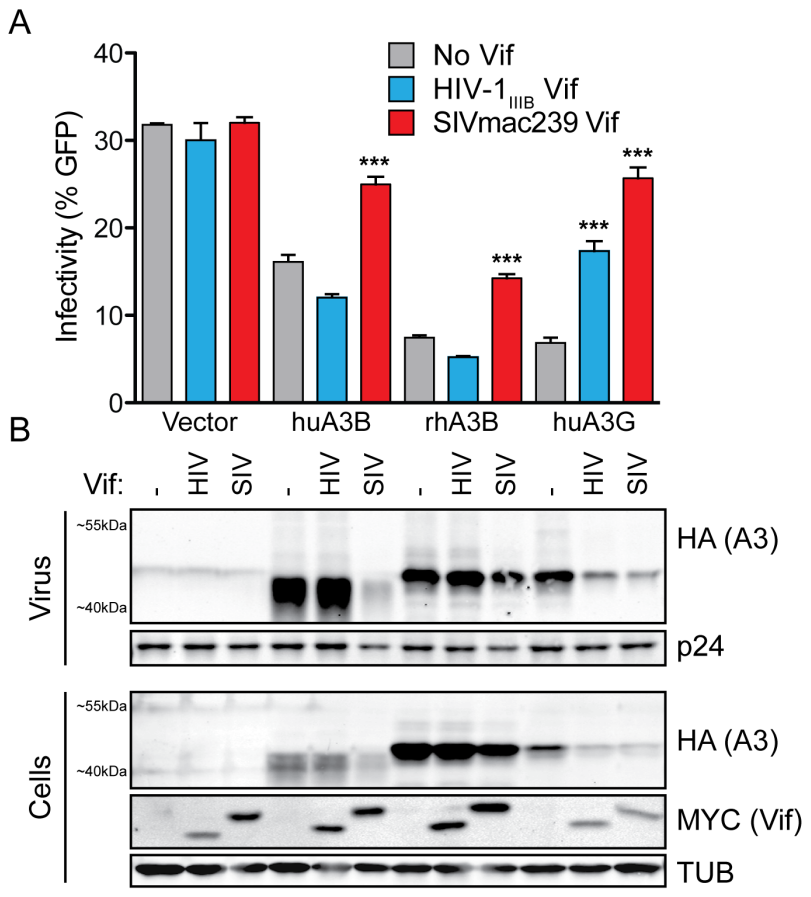

Figure 2: SIVmac239 Vif efficiently counteracts huA3B-mediated restriction of HIV-1. A. Bar graph depicting the infectivity (measured as \% infected CEM-GFP reporter) of Vif-deficient HIV-1 ${ }_{\text {ІІІв }}$ complemented with vector (grey bars), HIV-1 ${ }_{\text {IIIB }}$ Vif (blue bars), or SIVmac239 Vif (red bars); produced in the presence of vector control, huA3B, rhA3B or huA3G ( $n=3$; mean and SD shown). Asterisks indicate level of significance, compared to the no Vif condition $(* * * p<$ 0.001, as determined by one-way ANOVA). B. Representative immunoblots for each infection condition are shown beneath each histogram bar. Purified viral particles were blotted for HA to detect A3 and for p24 (Gag) as a loading control. Producer cell lysates were blotted for HA to detect A3, for MYC to detect Vif, and for Tubulin (TUB) as a loading control. The migration positions of molecular weight standards are indicated next to the anti-MYC (Vif) panels. 
We next asked whether the SLQ motif of Vif, which mediates interaction with ELOC of the E3 ubiquitin ligase complex, is important for mediating degradation of huA3B. HIV-1 $1_{\text {IIIB }}$ Vif and SIVmac239 Vif are only $30 \%$ identical at the amino acid level, but the SLQ motif is conserved (Fig. 3B). We transfected cells with APOBEC3 and Vif constructs as shown in Fig. 3C. Mutation of the SLQ region to AAA in HIV-1 ${ }_{\text {IIIB }}$ Vif abrogated its ability to mediate degradation of huA3G. Similarly, mutation of the SLQ region to AAA in SIVmac239 Vif also abolished degradation of huA3G (Fig. 3C). Neither wild-type nor SLQ- > AAA versions of HIV-1 ${ }_{\text {IIIB }}$ Vif caused degradation of huA3B. The SLQ- > AAA mutation in SIVmac239 Vif prevented Vif-mediated degradation of huA3B, indicating that this Vif protein interacts with the E3 ligase complex to degrade huA3B in a manner similar to the interaction of HIV-1 Vif and huA3G (Fig. 3C).

\section{SIVmac239 Vif rescues cells from huA3B- mediated cytotoxicity}

$\mathrm{HuA} 3 \mathrm{~B}$ is geno/cytotoxic in cell culture systems when overexpressed $[1,13,43]$. HuA3B localizes to the nucleus of cells, where it accesses genomic DNA and causes massive amounts of C-to-U deamination events. This leads to abasic sites, catastrophic levels of mutation, and ultimately cell death [1]. To determine if SIVmac239 Vif could save cells from huA3B-mediated cytotoxicity, we stably expressed huA3B-eGFP or eGFP alone under the control of a doxycycline-inducible promoter in T-REx 293 cells, allowing for titratable expression of the protein [1]. Vector control, HIV-1 ${ }_{\text {IIIB }}$ Vif, and SIVmac239 Vif were expressed stably in the inducible huA3B and GFP cells, and expression was confirmed by immunoblotting (inset images, Fig. 4A \& 4B). These cells were plated in increasing concentrations of doxycycline to assess viability in the constitutive presence of Vif and the inducible presence of huA3B.

The cells that inducibly expressed huA3B alone (i.e. huA3B + vector control) showed a marked decrease in viability, correlating with increased huA3B expression, with an $\mathrm{IC}_{50}$ value of $5 \times 10^{-1} \mathrm{pg} / \mathrm{mL}$ doxycycline (Fig. 4A). Stable expression of HIV-1 ${ }_{\text {IIIB }}$ Vif counteracted huA3B at an intermediate level, as demonstrated by significantly increased viability at $1-10^{3} \mathrm{pg} / \mathrm{mL}$ doxycycline, and increasing the $\mathrm{IC}_{50}$ value to $4.4 \times 10^{3}$ $\mathrm{pg} / \mathrm{mL}$ doxycycline. Additionally, as seen in immunoblots, there is a modest decrease in detected huA3B compared

A

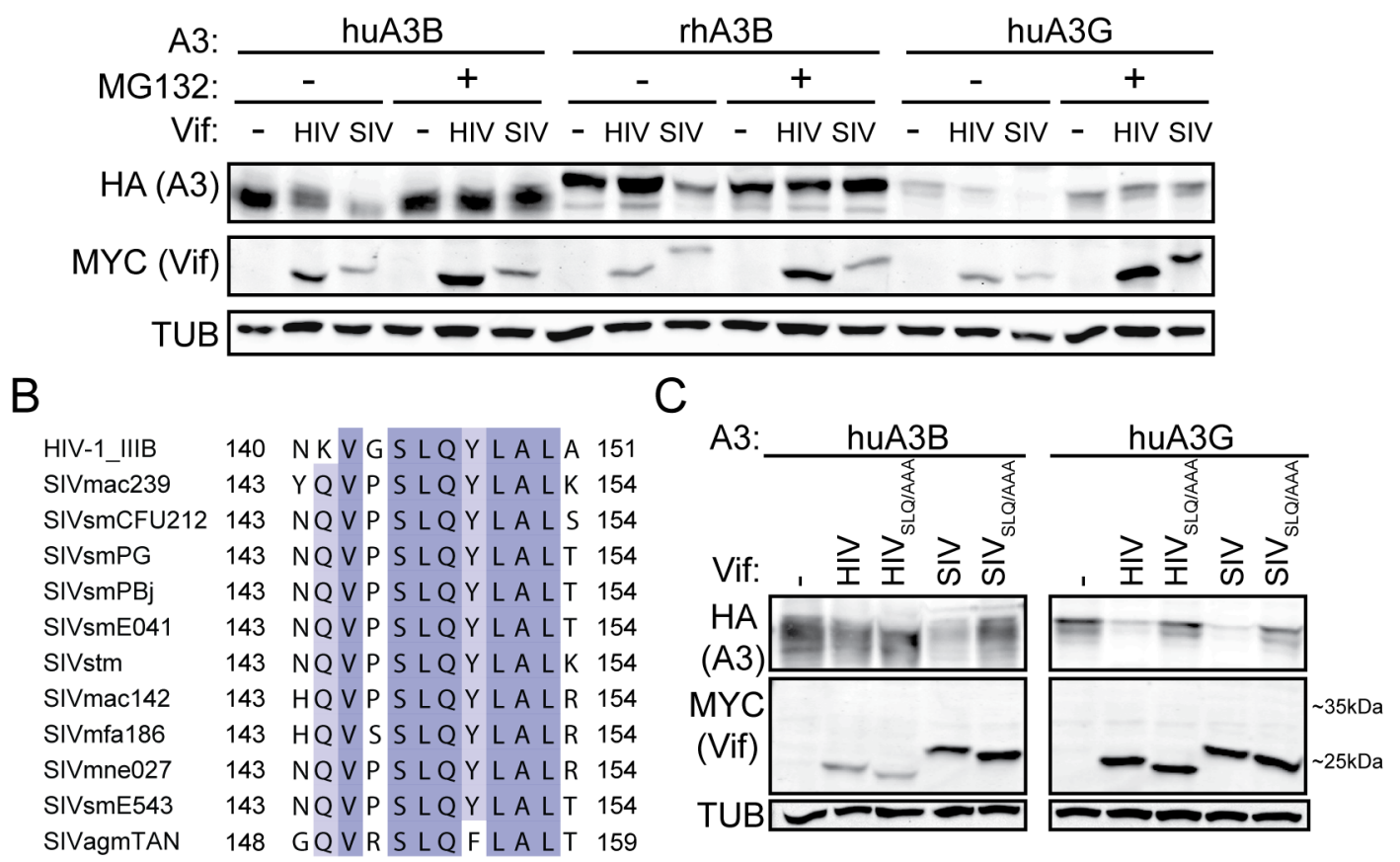

Figure 3: SIVmac239 Vif degradation of huA3B is analogous to HIV-1IIIB Vif degradation of huA3G. A. Immunoblots demonstrating inhibition of Vif-mediated degradation of A3 proteins in the presence of MG132 (5 $\mu \mathrm{M}, 16$ hours) or an equivalent amount of acetonitrile as a vehicle control. B. Amino acid alignment of the ELOC-binding SLQ region of the HIV-1 and SIV Vif proteins used in this study. The residues are shaded for conservation, with darker shades corresponding to more conserved positions. The residue positions included in the alignment are indicated. The conserved SLQ tri-residue motif is underlined. C. Immunoblots demonstrating that SIVmac239 Vif-mediated degradation of huA3B is dependent on the SLQ motif, as is HIV-1 ${ }_{\text {IIIB }}$ and SIVmac239 degradation of huA3G. Cell lysates were blotted for MYC to detect Vif, for HA to detect A3, and for tubulin (TUB) as a loading control. 
with no Vif (Fig. 4B). Stable expression of SIVmac239 Vif robustly counteracted huA3B and showed fully rescued or significantly increased levels of viability at all tested doxycycline concentrations. The maximal decrease in viability observed was only $30 \%$, preventing the determination of an $\mathrm{IC}_{50}$ value. The amount of huA3B detectable by immunoblot is only moderately less than that with no Vif, or HIV-1 ${ }_{\text {IIIB }}$, and still clearly detectable. These data indicate that SIVmac239 Vif may counteract huA3B through both a canonical degradation mechanism (likely the major pathway based on the aforementioned results) as well as, we speculate, a non-canonical mechanism such as cytoplasmic sequestration. This theory is not without precedent as HIV-1 Vif has been reported to alter the subcellular localization of APOBEC3 enzymes [37]. For comparison, cells that inducibly expressed GFP showed a constant level of viability with increasing GFP expression, regardless of co-expression of HIV-1 ${ }_{\text {IIIB }}$ Vif or SIV Vif (Fig. 4C \& 4D).

\section{SIVmac239 Vif degrades endogenous huA3B}

To begin to assess the feasibility of using SIVmac239 Vif or a derivative to counteract endogenous huA3B as an anti-cancer therapeutic, we examined the

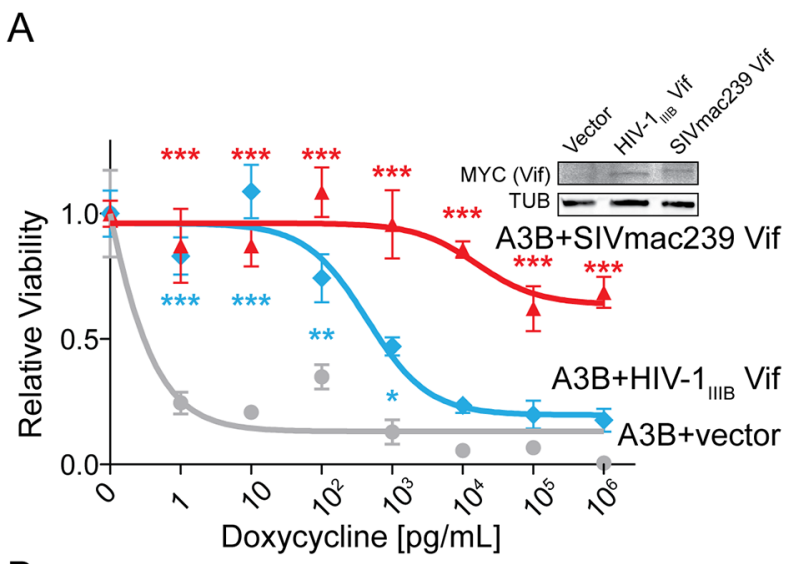

B

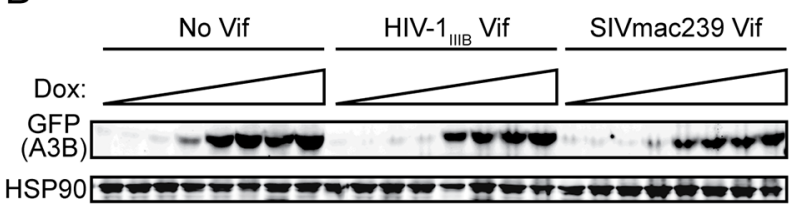

effect of SIVmac239 Vif in three cancer cell lines that endogenously express high levels of huA3B: HCC1569 cells, a human breast cancer cell line; JSQ3, a human head and neck cancer cell line; and OVCAR5, a human ovarian cancer cell line $[1,3]$. These three cell lines were transfected with HIV-1 ${ }_{\text {IIIB }}$ Vif and SIVmac239 Vif expression constructs, as well as empty vector. No significant difference in levels of endogenous huA3B was observed in cells stably expressing the vector control or HIV- ${ }_{\text {IIIB }}$ Vif (Fig. 5A \& 5B). In contrast, HCC1569, JSQ3, and OVCAR5 cells engineered to express SIVmac239 Vif all showed significantly lower levels of huA3B, indicating that SIVmac239 Vif is capable of mediating the degradation of endogenous huA3B in cancer cells (Fig. 5A \& 5B).

We further characterized SIVmac239 Vif-mediated degradation of endogenous huA3B in the OVCAR5 cell line. As shown with overexpressed huA3B, mutation of the SLQ region to AAA in SIVmac239 Vif abrogated its ability to mediate degradation of endogenous huA3B (Fig. 5C). Changing the SLQ region to AAA in HIV-1 Vif had no effect, as neither of these proteins mediated degradation of huA3B (Fig. 5C). In further agreement with the overexpression experiments, treatment of the OVCAR5 cells with the proteasomal inhibitor MG132 rescued

C

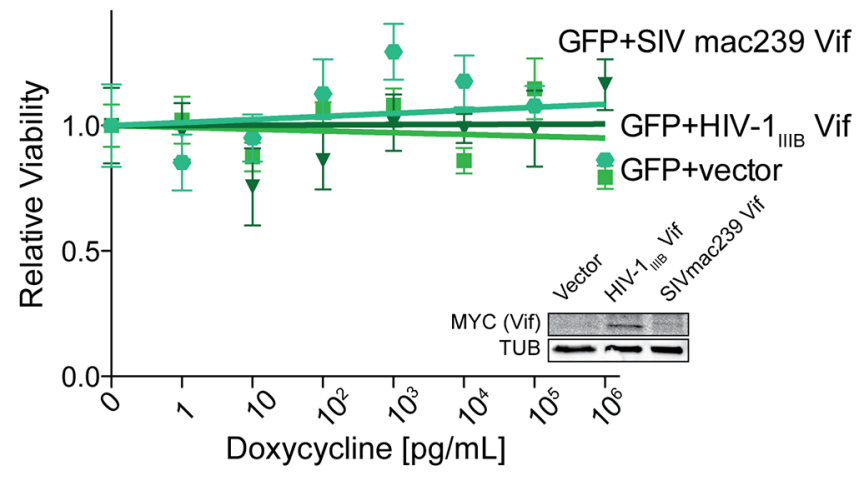

D

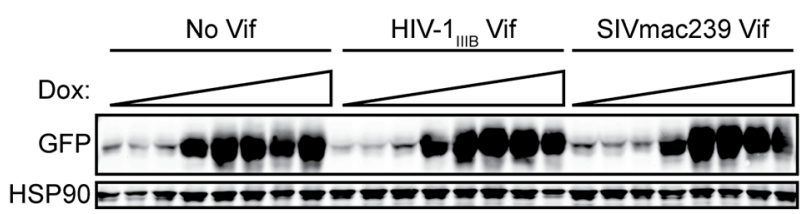

Figure 4: SIVmac239 Vif rescues cells from huA3B-mediated DNA damage and cytotoxicity. A. Clonogenic assay for T-REx 293 cells expressing huA3B-eGFP with doxycycline induction, and stably expressing vector (grey), HIV-1 ${ }_{\text {IIIB }}$ Vif (blue), or SIVmac239 Vif (red). Relative viability indicates the ratio of clones that grew in increasing doxycycline, compared to no doxycycline induction $(n=3$, mean and SD shown). The lysates (inset) were blotted for MYC to detect Vif and tubulin (TUB) as a loading control. Asterisks indicate level of significance, compared to vector condition $\left({ }^{*} p<0.05 ; * *<0.01 ; * * p<0.001\right.$, as determined by two-way ANOVA). B. Representative immunoblots for cells at each doxycycline (dox) concentration show induction of huA3B in the presence of the indicated Vif constructs. Cell lysates were blotted for GFP to detect huA3B-eGFP and for HSP90 as a loading control. C. Clonogenic assay for T-REx 293 cells

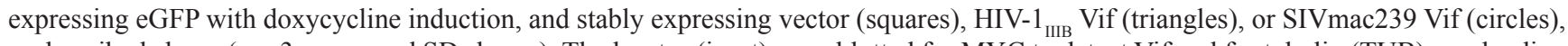
as described above ( $n=3$, mean and SD shown). The lysates (inset) were blotted for MYC to detect Vif and for tubulin (TUB) as a loading control. D. Representative immunoblots for cells at each doxycycline (dox) concentration show induction of GFP in the presence of the indicated Vif constructs. The lysates were blotted for GFP and for HSP90 as a loading control. 
endogenous huA3B from SIVmac239 Vif-mediated degradation (Fig. 5C). Based on the established mechanism for Vif function, these data indicate that SIVmac239 Vif interacts with ELOC of the E3 ubiquitin ligase complex via its SLQ motif to mediate proteasomal degradation of endogenous huA3B.

\section{A}

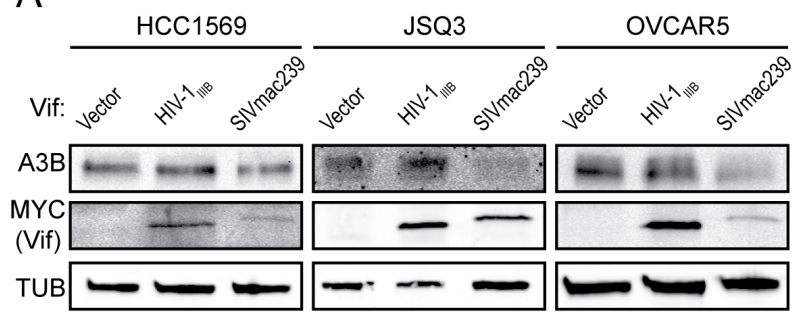

B

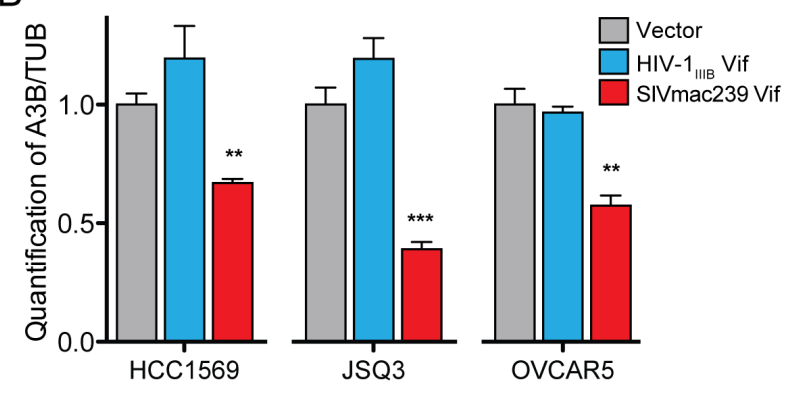

C

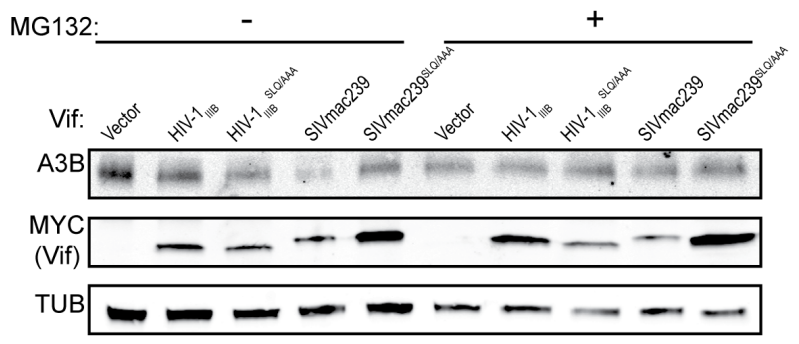

Figure 5: SIVmac239 Vif degrades endogenous huA3B in cancer cells. A. Representative immunoblots of HCC1569, JSQ3, and OVCAR5 cells expressing empty vector, HIV-1 ${ }_{\text {IIIB }}$ Vif, or SIVmac239 Vif. Cell lysates were blotted for endogenous huA3B, for MYC to detect Vif, and for tubulin (TUB) as a loading control. B. Quantification of endogenous huA3B in cancer cells expressing empty vector, HIV-1 ${ }_{\text {IIIB }}$ Vif, or SIVmac239 Vif. A3B levels were normalized to Tubulin, and the amount of A3B in the presence of vector, for each cell line, was set at $1(n=3$, mean and $\mathrm{SD}$ shown). Asterisks indicate level of significance, compared to vector control $(* * p<0.01 ; * * * p<0.001$, as determined by two-way ANOVA). C. Representative immunoblot of OVCAR5 cells demonstrating that SIVmac239 Vif-mediated degradation of endogenous huA3B is dependent on the SLQ motif and that this degradation is inhibited in the presence of MG132 $(5 \mu \mathrm{M}$, 16 hours). Cell lysates were blotted for endogenous huA3B, for MYC to detect Vif, and for tubulin (TUB) as a loading control.

\section{DISCUSSION}

A3B contributes to genomic mutation in breast and other cancers, and associates with poor patient outcomes (reviewed in [16-18]). As such, it would be beneficial to cancer research and patient therapy to be able to counteract this potent DNA mutator. A3B is part of a larger family of APOBEC3 proteins, some of which have physiologic functions in restricting HIV-1 replication, and these are counteracted by HIV-1 Vif (reviewed in $[19,20])$. HuA3B is not efficiently degraded by HIV-1 Vif [21-28], however, HIV-1 Vif may bind huA3B as indicated by co-immunoprecipitation studies [21,37]. We hypothesized that the Vif protein from another lentivirus may be capable of counteracting huA3B, as diverse lentiviruses are restricted by differing subsets of the host's APOBEC 3 proteins. By surveying a panel of Vif proteins from lentiviruses that infect different animal hosts, we identified SIVmac239 Vif as a potent inhibitor of both overexpressed and endogenously upregulated huA3B, degrading huA3B in an analogous manner to HIV- $1_{\text {IIIB }}$ Vif mediating degradation of huA3G.

In testing the conservation of the ability of Vif proteins from various SIV strains to degrade huA3B, we found that many but not all SIV Vif proteins share this trait. Such an ability to degrade huA3B is potentially due to structural and functional conservation of huA3B with simian APOBEC3 proteins and the ability of some of these simian enzymes to restrict the relevant SIV strains, thus being legitimate targets of the respective Vif ubiquitin ligase complexes. For example, rhA3B has been demonstrated here and in other studies to be counteracted by SIVmac Vif, suggesting that this protein may be relevant for SIVmac infection of rhesus macaques [22, 32]. In contrast, HIV-1 is not restricted by huA3B in T cells, the major cellular target of HIV-1, and HIV-1 Vif does not efficiently counteract huA3B [21-28]. These species specificities can be utilized to further understand the relevant protein interaction surfaces. For instance, the ability of HIV-1 Vif to counteract huA3F but not rhA3F has led to better understanding to this protein interaction surface [32, 44-46]. By better understanding the interaction between huA3B and SIVmac239 Vif, SIVmac239 Vif may be engineered to become more efficient at degrading huA3B and to increase its specificity for huA3B over other APOBEC3 proteins, resulting in a better molecular probe and cancer therapeutic.

Interestingly, although we clearly showed that SIVmac239 Vif is capable of degrading huA3B, it also appeared capable of neutralizing the cytotoxic effects of huA3B beyond the canonical degradation mechanism. Based on prior studies [37], we hypothesize that SIVmac239 Vif may also bind huA3B in the cytosol and prevent it from importing into the nuclear compartment. 
Such a sequestration mechanism could help protect genomic DNA from huA3B's genotoxic activities, as has been inferred for the related APOBEC3A (A3A) protein $[47,48]$. However, this theory needs extensive testing for validation and to distinguish it from other possible mechanisms. HIV-1 $1_{\text {IIIB }}$ Vif was also able to mediate neutralization of huA3B, albeit at a much lower level than SIVmac239 Vif. This may be related to binding rather than degradation of the protein. This observation also suggests that HIV-1 Vif could protect cells from huA3B's oncogenic effects, and that HIV-1 infected individuals may be partly protected from the onset or progression of some malignancies. However, this does not seem to be the case as HIV-1 infection is associated with an increased risk of several AIDS-defining and non-AIDS defining cancers $[49,50]$.

This study is the first to show that SIVmac239 Vif can degrade the genomic DNA mutator huA3B in living cells. Degradation of endogenous huA3B was observed in cell lines representing three different types of human cancer, suggesting that although cellular factors that regulate huA3B are not yet elucidated, this finding may have implications for treating a wide range of huA3B effected malignancies. The data presented here have strong implications for developing SIVmac239 Vif as a molecular tool for future studies on the mutagenic properties of huA3B, and for neutralizing huA3B in cancer to halt tumor mutagenesis, prevent therapy resistance, and improve the treatment and prognosis of cancer patients.

\section{MATERIALS AND METHODS}

\section{APOBEC3 expression constructs}

The APOBEC3 proteins huA3B (GenBank accession no. NM004900), huA3G (GenBank NM021822), and rhA3B (GenBank JF714485, but with the asparagine at amino acid residue 316 restored to aspartate [51]) were expressed with carboxy-terminal HA tag in the pcDNA3.1(+) vector (Invitrogen). cDNA was provided by Dr. Theodora Hatziioannou (Aaron Diamond AIDS Research Center, New York) [32]. Additionally, huA3B was expressed with a carboxy-terminal eGFP tag in the doxycycline-inducible pcDNA5TO vector (Clontech).

\section{Vif nomenclature and expression constructs}

Each Vif protein is described by virus type (HIV, SIV, etc) and a strain/isolate identifier (IIIB, mac239, etc) according to standard conventions in the retrovirus field. The lentiviral Vif proteins from HIV-1 ${ }_{\text {IIIB }}$ (protein sequence matches GenBank EU541617),

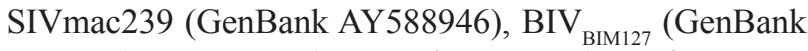
M32690), MVV ${ }_{1514}$ (GenBank M60610), and FIV (GenBank m25381) were codon optimized (GenScript Corporation) and expressed with a carboxy-terminal MYC tag in the pVR1012 vector [29]. Vif expression constructs from SIVsmCFU212 (GenBank JX860407), SIVsmPG (GenBank AAC68657), SIVsmPBj (GenBank AAB22996), SIVsmE041 (GenBank HM059825), SIVstm (GenBank AAA91941), SIVmac142 (GenBank Y00277), SIVmfa186 (GenBank KF030930), SIVmne027 (GenBank U70412), SIVsmE543-3 (GenBank U72748), and SIVagmTAN (GenBank AAC57053) were derived originally in the Johnson lab (Boston College) [38]. These cDNAs were subcloned into the pVR1012 vector with a carboxyterminal MYC tag. The Vif expression construct pVR1012 was a generous gift of Dr. Xiao-Fang Yu (John Hopkins, Baltimore). For transient expression in HCC1569 and JSQ3 cells, the constructs were transfected with TransIT-2020 (Mirus) and TransIT-X2 (Mirus), respectively. For stable expression in OVCAR5 cells, HIV-1 ${ }_{\text {IIIB }}$ and SIVmac239 Vif were subcloned into the pLenti4-Hygro-TO backbone, transduced into OVCAR5 cells, and a stably expressing pool was selected with hygromycin.

\section{HIV constructs}

The Vif proficient and Vif deficient (X26X27) HIV-1 ${ }_{\text {IIIB }}$ A200C proviral expression constructs (GenBank EU541617) have been reported [52].

\section{Cell lines}

293T cells, T-REx 293 (Invitrogen) cells, and JSQ3 cells were maintained in Dulbecco's modified Eagle medium (DMEM) containing 10\% fetal bovine serum (FBS) and $0.5 \%$ penicillin-streptomycin (P/S). CEMGFP, HCC1569, and OVCAR5 cells were maintained in RPMI medium with $10 \% \mathrm{FBS}$ and $0.5 \% \mathrm{P} / \mathrm{S}$. The CEMGFP HIV reporter cell line was obtained from the NIH AIDS Reagent Program [53]; the breast cancer cell line HCC1569 from ATCC; the head and neck cancer cell line JSQ3 from Dr. Mark Herzberg (University of Minnesota); and the ovarian cancer cell line OVCAR5 from the Mayo Clinic ovarian cell line repository.

\section{Immunoblotting}

Cell lysates were prepared by resuspending washed cell pellets directly in $2.5 \mathrm{X}$ Laemmli sample buffer. Viral particles were purified from the filtered supernatant by centrifugation prior to resuspension in $2.5 \mathrm{X}$ Laemmli sample buffer. A3-HA was detected with monoclonal mouse anti-HA (BioLegend), Vif-MYC was detected with polyclonal rabbit anti-MYC (Sigma-Aldrich), Tubulin (TUB) was detected with monoclonal mouse anti- $\alpha$-Tubulin (Covance), HIV-1 Gag was detected with monoclonal mouse anti-HIV-1 p24 (NIH AIDS Reagent Program) [54], A3-GFP was detected with monoclonal mouse anti-GFP (Clontech), HSP90 was detected with mouse anti-HSP90 (BD Biosciences). A3B was detected 
with rabbit monoclonal anti-A3B [55] (Brown et al., in prep). To determine endogenous huA3B degradation, the huA3B and Tubulin bands were quantified from immunoblots using ImageJ (1.42q), and huA3B levels were normalized to those of Tubulin. These values were analyzed using a two-way ANOVA. Bonferroni's method for post-hoc testing was used to compare the amount of huA3B in the presence of vector, HIV-1 ${ }_{\text {IIIB }}$ Vif, and SIVmac239 Vif. Statistical analyses were done with Prism 5 (GraphPad Software Inc.).

\section{Vif degradation}

$293 \mathrm{~T}$ cells were transfected in triplicate with pVR1020-Vif-MYC or empty vector, at levels normalized by immunoblot, and pcDNA3.1 A3-HA, or empty vector, as indicated, using PEI (polyethyleneimine; Polysciences, Inc.). The following amounts of Vif expression construct were transfected for Fig. 1A: HIV-1 ${ }_{\text {IIIB }} 50-200 \mathrm{ng}$; SIVmac239 50-200 ng; BIV 100-400 ng; FIV 50-200 ng; MVV 100-400 ng. For Fig. 1B, the following amounts of Vif expression construct were transfected: HIV-1 ${ }_{\text {IIIB }}$ 50-100 ng; SIVmac239 100-200 ng; SIVsmCFU212 200-400 ng; SIVsmPG 200-400 ng; SIVsmPBj 200-400 ng; SIVsmE041 200-400 ng; SIVstm 400-800 ng; SIVmac142 200-400 ng; SIVmfa186 200-400 ng; SIVmne027 200-400 ng, SIVsmE543 200-400 ng; SIVagmTAN 400-800 ng. After 48 hours, the cells were harvested for immunoblot analysis. To inhibit proteasomal degradation, MG132 (American Peptide) was added at $5 \mu \mathrm{M}, 16$ hours before harvesting the cells.

\section{HIV-1 single cycle infection with replication- proficient virus}

The single-cycle infectivity assays were performed as previously reported [22] by transfecting 293T cells (TransIT-LT1; Mirus) in triplicate with $1 \mu \mathrm{g}$ of a Vifdeficient HIV-1 proviral expression construct along with $25 \mathrm{ng}$ of A3-HA expression construct or empty vector, and $25 \mathrm{ng}$ of Vif-MYC expression construct or empty vector. After 48 hours, purified virus-containing supernatants were used to infect the CEM-GFP HIV-1 reporter cells, and cell and viral particle lysates were prepared for immunoblotting. Infectivity was analyzed using a one-way ANOVA. Dunnett's method for post-hoc testing was used to compare increases in infectivity in the presence of the Vif expression constructs with vector control. Statistical analyses were done with Prism 5 (GraphPad Software Inc.).

\section{Flow cytometry}

HIV-infected CEM-GFP cells were prepared for flow cytometry by fixation in 4\% paraformaldehyde. GFP fluorescence was measured on a BD FACS Canto II flow cytometer (BD Biosciences). All data were analyzed using Flow Jo flow cytometry analysis software (version 8.8.7). GFP fluorescence was quantified from gated live cell populations.

\section{Viability assay}

T-REx 293 cells (Invitrogen), which stably express the tetracycline repressor, were transfected with pcDNA5TO-A3B-eGFP and pcDNA5TO-eGFP constructs using TransIT-LT1 (Mirus). Stable clones were selected with hygromycin and blasticidin. These T-REx 293 huA3B and T-REx 293 GFP stable clones were further engineered to stably express HIV-1 ${ }_{\text {IIIB }}$ Vif-myc, SIVmac239 VifMYC or vector by transfection of pcDNA3.1 expression constructs and selection with G418. To assess viability, equal numbers of cells were plated in triplicate in increasing doxycycline concentrations and clones were allowed to form. The clones were quantified using ImageJ (1.42q) software. In parallel, these cells were plated in increasing doxycycline concentrations and harvested after 48 hours for immunoblotting. Viability data were analyzed using a two-way ANOVA. Bonferroni's method for posthoc testing was used to compare viability in the presence of vector control to the viability with HIV-1 ${ }_{\text {IIIB }}$ Vif and SIVmac239 Vif. Statistical analyses and TCID ${ }_{50}$ were done with Prism 5 (GraphPad Software Inc.).

\section{ACKNOWLEDGMENTS AND FUNDING}

We thank B.D. Anderson, M.A. Carpenter, and B. Leonard for comments. The following reagents were obtained through the NIH AIDS Reagent Program, Division of AIDS, NIAID, NIH: HIV-1 p24 Monoclonal Antibody (183-H12-5C) from Drs. B. Chesebro and K. Wehrly and CEM-GFP from Dr. J. Corbeil. RhA3B cDNA was a generous gift from Dr. T. Hatziioannou (Aaron Diamond AIDS Research Center, New York), the pVR1012 expression construct from Dr. X.-F. Yu (John Hopkins, Baltimore), and the head/neck cell line from Dr. M. Herzberg (University of Minnesota). Salary support for AML was provided in part by subaward 7433sc from NIH P50-GM082250.

These studies were supported by NIH grants to WEJ (NIAID R01 AI095092 and AI083118) and RSH (NIAID R01 AI064046 and NIGMS P01 GM091743).

\section{CONFLICTS OF INTEREST}

RSH is a cofounder of ApoGen Biotechnologies Inc. The other authors have no conflicts of interest. 


\section{REFERENCES}

1. Burns MB, Lackey L, Carpenter MA, Rathore A, Land AM, Leonard B, Refsland EW, Kotandeniya D, Tretyakova N, Nikas JB, Yee D, Temiz NA, Donohue DE, McDougle RM, Brown WL, Law EK, et al. APOBEC3B is an enzymatic source of mutation in breast cancer. Nature. 2013; 494:366-370.

2. Burns MB, Temiz NA, Harris RS. Evidence for APOBEC3B mutagenesis in multiple human cancers. Nat Genet. 2013; 45:977-983.

3. Leonard B, Hart SN, Burns MB, Carpenter MA, Temiz NA, Rathore A, Vogel RI, Nikas JB, Law EK, Brown WL, Li Y, Zhang Y, Maurer MJ, Oberg AL, Cunningham JM, Shridhar V, et al. APOBEC3B upregulation and genomic mutation patterns in serous ovarian carcinoma. Cancer Res. 2013; 73:7222-7231.

4. Alexandrov LB, Nik-Zainal S, Wedge DC, Aparicio SA, Behjati S, Biankin AV, Bignell GR, Bolli N, Borg A, Borresen-Dale AL, Boyault S, Burkhardt B, Butler AP, Caldas C, Davies HR, Desmedt C, et al. Signatures of mutational processes in human cancer. Nature. 2013; 500:415-421.

5. Roberts SA, Lawrence MS, Klimczak LJ, Grimm SA, Fargo D, Stojanov P, Kiezun A, Kryukov GV, Carter SL, Saksena G, Harris S, Shah RR, Resnick MA, Getz G, Gordenin DA. An APOBEC cytidine deaminase mutagenesis pattern is widespread in human cancers. Nat Genet. 2013; 45:970-976.

6. Nik-Zainal S, Alexandrov LB, Wedge DC, Van Loo P, Greenman CD, Raine K, Jones D, Hinton J, Marshall J, Stebbings LA, Menzies A, Martin S, Leung K, Chen L, Leroy C, Ramakrishna $\mathrm{M}$, et al. Mutational processes molding the genomes of 21 breast cancers. Cell. 2012; 149:979-993.

7. Lawrence MS, Stojanov P, Polak P, Kryukov GV, Cibulskis K, Sivachenko A, Carter SL, Stewart C, Mermel CH, Roberts SA, Kiezun A, Hammerman PS, McKenna A, Drier Y, Zou L, Ramos AH, et al. Mutational heterogeneity in cancer and the search for new cancer-associated genes. Nature. 2013; 499:214-218.

8. Cancer Genome Atlas Research Network. Comprehensive molecular characterization of urothelial bladder carcinoma. Nature. 2014; 507:315-322.

9. Rebhandl S, Huemer M, Gassner FJ, Zaborsky N, Hebenstreit D, Catakovic K, Grossinger EM, Greil R, Geisberger R. APOBEC3 signature mutations in chronic lymphocytic leukemia. Leukemia. 2014; 28:1929-1932.

10. Henderson S, Chakravarthy A, Su X, Boshoff C, Fenton TR. APOBEC-mediated cytosine deamination links PIK3CA helical domain mutations to human papillomavirus-driven tumor development. Cell Rep. 2014; 7:1833-1841.

11. Waters CE, Saldivar JC, Amin ZA, Schrock MS, Huebner K. FHIT loss-induced DNA damage creates optimal APOBEC substrates: Insights into APOBEC-mediated mutagenesis. Oncotarget. 2015; 6:3409-3419.
12. Roberts SA, Sterling J, Thompson C, Harris S, Mav D, Shah R, Klimczak LJ, Kryukov GV, Malc E, Mieczkowski PA, Resnick MA, Gordenin DA. Clustered mutations in yeast and in human cancers can arise from damaged long single-strand DNA regions. Mol Cell. 2012; 46:424-435.

13. Taylor BJ, Nik-Zainal S, Wu YL, Stebbings LA, Raine K, Campbell PJ, Rada C, Stratton MR, Neuberger MS. DNA deaminases induce break-associated mutation showers with implication of APOBEC3B and 3A in breast cancer kataegis. Elife. 2013; 2:e00534.

14. Sieuwerts AM, Willis S, Burns MB, Look MP, MeijerVan Gelder ME, Schlicker A, Heideman MR, Jacobs H, Wessels L, Leyland-Jones B, Gray KP, Foekens JA, Harris RS, Martens JW. Elevated APOBEC3B correlates with poor outcomes for estrogen-receptor-positive breast cancers. Horm Cancer. 2014; 5:405-413.

15. Cescon DW, Haibe-Kains B, Mak TW. APOBEC3B expression in breast cancer reflects cellular proliferation, while a deletion polymorphism is associated with immune activation. Proc Natl Acad Sci U S A. 2015; 112:2841-2846.

16. Swanton C, McGranahan N, Starrett GJ, Harris RS. APOBEC Enzymes: Mutagenic Fuel for Cancer Evolution and Heterogeneity. Cancer Discov. 2015; 5:704-712.

17. Henderson S, Fenton T. APOBEC3 genes: retroviral restriction factors to cancer drivers. Trends Mol Med. 2015; 21:274-284.

18. Roberts SA, Gordenin DA. Hypermutation in human cancer genomes: footprints and mechanisms. Nat Rev Cancer. 2014; 14:786-800.

19. Harris RS, Dudley JP. APOBECs and virus restriction. Virology. 2015; 479-480C:131-145.

20. Desimmie BA, Delviks-Frankenberrry KA, Burdick RC, Qi D, Izumi T, Pathak VK. Multiple APOBEC3 Restriction Factors for HIV-1 and One Vif to Rule Them All. J Mol Biol. 2014; 426:1220-1245.

21. Baig TT, Feng Y, Chelico L. Determinants of efficient degradation of APOBEC3 restriction factors by HIV-1 Vif. J Virol. 2014; 88:14380-14395.

22. Hultquist JF, Lengyel JA, Refsland EW, LaRue RS, Lackey L, Brown WL, Harris RS. Human and rhesus APOBEC3D, APOBEC3F, APOBEC 3G, and APOBEC3H demonstrate a conserved capacity to restrict Vif-deficient HIV-1. J Virol. 2011; 85:11220-11234.

23. Shirakawa K, Takaori-Kondo A, Kobayashi M, Tomonaga M, Izumi T, Fukunaga K, Sasada A, Abudu A, Miyauchi Y, Akari H, Iwai K, Uchiyama T. Ubiquitination of APOBEC3 proteins by the Vif-Cullin5-ElonginBElonginC complex. Virology. 2006; 344:263-266.

24. Doehle BP, Schafer A, Cullen BR. Human APOBEC3B is a potent inhibitor of HIV-1 infectivity and is resistant to HIV-1 Vif. Virology. 2005; 339:281-288.

25. Yu Q, Chen D, Konig R, Mariani R, Unutmaz D, Landau NR. APOBEC3B and APOBEC3C are potent 
inhibitors of simian immunodeficiency virus replication. J Biol Chem. 2004; 279:53379-53386.

26. Zhou T, Han Y, Dang Y, Wang X, Zheng YH. A novel HIV-1 restriction factor that is biologically distinct from APOBEC3 cytidine deaminases in a human $\mathrm{T}$ cell line CEM.NKR. Retrovirology. 2009; 6:31.

27. Rose KM, Marin M, Kozak SL, Kabat D. Regulated production and anti-HIV type 1 activities of cytidine deaminases APOBEC3B, 3F, and 3G. AIDS Res Hum Retroviruses. 2005; 21:611-619.

28. Bishop KN, Holmes RK, Sheehy AM, Davidson NO, Cho SJ, Malim MH. Cytidine deamination of retroviral DNA by diverse APOBEC proteins. Curr Biol. 2004; 14:1392-1396.

29. LaRue RS, Lengyel J, Jónsson SR, Andrésdóttir V, Harris RS. Lentiviral Vif degrades the APOBEC3Z3/ APOBEC3H protein of its mammalian host and is capable of cross-species activity. J Virol. 2010; 84:8193-8201.

30. Wang J, Zhang W, Lv M, Zuo T, Kong W, Yu X. Identification of a Cullin5-ElonginB-ElonginC E3 complex in degradation of feline immunodeficiency virus Vif-mediated feline APOBEC3 proteins. J Virol. 2011; 85:12482-12491.

31. Zhang W, Wang H, Li Z, Liu X, Liu G, Harris RS, Yu XF. Cellular requirements for bovine immunodeficiency virus Vif-mediated inactivation of bovine APOBEC3 proteins. J Virol. 2014; 88:12528-12540.

32. Virgen CA, Hatziioannou T. Antiretroviral activity and Vif sensitivity of rhesus macaque APOBEC3 proteins. J Virol. 2007; 81:13932-13937.

33. LaRue RS, Andresdottir V, Blanchard Y, Conticello SG, Derse D, Emerman M, Greene WC, Jonsson SR, Landau NR, Lochelt M, Malik HS, Malim MH, Munk C, O'Brien SJ, Pathak VK, Strebel K, et al. Guidelines for naming nonprimate APOBEC3 genes and proteins. J Virol. 2009; 83:494-497.

34. Munk C, Beck T, Zielonka J, Hotz-Wagenblatt A, Chareza S, Battenberg M, Thielebein J, Cichutek K, Bravo IG, O'Brien SJ, Lochelt M, Yuhki N. Functions, structure, and read-through alternative splicing of feline APOBEC3 genes. Genome Biol. 2008; 9:R48.

35. LaRue RS, Jónsson SR, Silverstein KA, Lajoie M, Bertrand D, El-Mabrouk N, Hotzel I, Andrésdóttir V, Smith TP, Harris RS. The artiodactyl APOBEC3 innate immune repertoire shows evidence for a multi-functional domain organization that existed in the ancestor of placental mammals. BMC Mol Biol. 2008; 9:104.

36. Smith JL, Pathak VK. Identification of specific determinants of human APOBEC3F, APOBEC3C, and APOBEC3DE and African green monkey APOBEC3F that interact with HIV-1 Vif. J Virol. 2010; 84:12599-12608.

37. Marin M, Golem S, Rose KM, Kozak SL, Kabat D. Human immunodeficiency virus type 1 Vif functionally interacts with diverse APOBEC3 cytidine deaminases and moves with them between cytoplasmic sites of mRNA metabolism. J Virol. 2008; 82:987-998.

38. Krupp A, McCarthy KR, Ooms M, Letko M, Morgan JS, Simon V, Johnson WE. APOBEC3G polymorphism as a selective barrier to cross-species transmission and emergence of pathogenic SIV and AIDS in a primate host. PLoS Pathog. 2013; 9:e1003641.

39. Mariani R, Chen D, Schrofelbauer B, Navarro F, Konig R, Bollman B, Munk C, Nymark-McMahon H, Landau NR. Species-specific exclusion of APOBEC3G from HIV-1 virions by Vif. Cell. 2003; 114:21-31.

40. Gaur R, Strebel K. Insights into the dual activity of SIVmac239 Vif against human and African green monkey APOBEC3G. PLoS One. 2012; 7:e48850.

41. Conticello SG, Harris RS, Neuberger MS. The Vif protein of HIV triggers degradation of the human antiretroviral DNA deaminase APOBEC3G. Curr Biol. 2003; 13:2009-2013.

42. Liu B, Yu X, Luo K, Yu Y, Yu XF. Influence of primate lentiviral Vif and proteasome inhibitors on human immunodeficiency virus type 1 virion packaging of APOBEC3G. J Virol. 2004; 78:2072-2081.

43. Shinohara M, Io K, Shindo K, Matsui M, Sakamoto T, Tada K, Kobayashi M, Kadowaki N, Takaori-Kondo A. APOBEC3B can impair genomic stability by inducing base substitutions in genomic DNA in human cells. Sci Rep. 2012; 2:806.

44. Zennou V, Bieniasz PD. Comparative analysis of the antiretroviral activity of $\mathrm{APOBEC} 3 \mathrm{G}$ and $\mathrm{APOBEC} 3 \mathrm{~F}$ from primates. Virology. 2006; 349:31-40.

45. Albin JS, LaRue RS, Weaver JA, Brown WL, Shindo K, Harjes E, Matsuo H, Harris RS. A single amino acid in human APOBEC3F alters susceptibility to HIV-1 Vif. J Biol Chem. 2010; 285:40785-40792.

46. Land AM, Shaban NM, Evans L, Hultquist JF, Albin JS, Harris RS. APOBEC3F determinants of HIV-1 Vif sensitivity. J Virol. 2014; 88:12923-12927.

47. Landry S, Narvaiza I, Linfesty DC, Weitzman MD. APOBEC3A can activate the DNA damage response and cause cell-cycle arrest. EMBO Rep. 2011; 12:444-450.

48. Land AM, Law EK, Carpenter MA, Lackey L, Brown WL, Harris RS. Endogenous APOBEC3A DNA cytosine deaminase is cytoplasmic and nongenotoxic. J Biol Chem. 2013; 288:17253-17260.

49. Engels EA, Biggar RJ, Hall HI, Cross H, Crutchfield A, Finch JL, Grigg R, Hylton T, Pawlish KS, McNeel TS, Goedert JJ. Cancer risk in people infected with human immunodeficiency virus in the United States. Int J Cancer. 2008; 123:187-194.

50. Grulich AE, van Leeuwen MT, Falster MO, Vajdic CM. Incidence of cancers in people with HIV/AIDS compared with immunosuppressed transplant recipients: a meta-analysis. Lancet. 2007; 370:59-67. 
51. McDougle RM, Hultquist JF, Stabell AC, Sawyer SL, Harris RS. D316 is critical for the enzymatic activity and HIV-1 restriction potential of human and rhesus APOBEC3B. Virology. 2013; 441:31-39.

52. Haché G, Shindo K, Albin JS, Harris RS. Evolution of HIV-1 isolates that use a novel Vif-independent mechanism to resist restriction by human APOBEC3G. Curr Biol. 2008; 18:819-824.

53. Gervaix A, West D, Leoni LM, Richman DD, WongStaal F, Corbeil J. A new reporter cell line to monitor HIV infection and drug susceptibility in vitro. Proc Natl Acad Sci U S A. 1997; 94:4653-4658.
54. Chesebro B, Wehrly K, Nishio J, Perryman S. Macrophagetropic human immunodeficiency virus isolates from different patients exhibit unusual V3 envelope sequence homogeneity in comparison with T-cell-tropic isolates: definition of critical amino acids involved in cell tropism. J Virol. 1992; 66:6547-6554.

55. Leonard B, McCann JL, Starrett GJ, Kosyakovsky L, Luengas EM, Molan AM, Burns MB, McDougle RM, Parker PJ, Brown WL, Harris RS. The PKC/NF- $\mathrm{BB}$ signalling pathway induces APOBEC3B expression in multiple human cancers. Cancer Res. 2015; 75:1-10. 